\section{THU0368 THE IMPACT OF PROFESSIONAL ACTIVITY IN ANKYLOSING SPONDYLITIS}

ANDRONACHE IULIA ${ }^{1}$, Victoria Cristina Suta ${ }^{2,3}$, Sabina Ciocodei ${ }^{3}$, Maria Suta ${ }^{3}$.

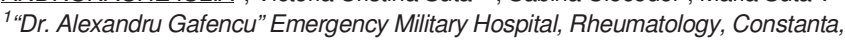
Romania; ${ }^{2}$ Sf. Apostol Andrei" Emergency Clinical County Hospital Constanta, 2nd Internal Medicine Clinic-, Constanta, Romania; ${ }^{3}$ Faculty of Medicine, "Ovidius" University Constanța, Constanta, Romania

Background: The mechanical stress due to strenuous professional activity cannot be easily quantified, yet it may lead to a wide range of musculoskeletal complaints. In ankylosing spondylitis (AS) patients mechanic stress may lead to the occurrence and the aggravation of enthesitis (1) and a profession involving an intense physical strain is correlated with the radiologic progression (2). While studies regarding the professional activities in ankylosing spondylitis patients are focused on the disease's impact on the work capacity, the type of labor may also influence the disease progression (3).

Objectives: To assess the relationship between the professional activity in AS patients, disease activity and radiological changes.

Methods: We enrolled 193 patients with AS admitted in the Rheumatology Department of the "Sf. Apostol Andrei" Emergency Clinical County Hospital. Patient evaluation was performed by a rheumatologist. All the data obtained from the medical history, clinical examination, laboratory tests and imaging studies was recorded at the same date. Data regarding the professional history, type of labor involved was also recorded. The physical strain due to professional activity was done using the methodology established by the National Institute for Medical Expertise and Recovery of Work Capacity which takes into account the amount of energy used in order to perform certain tasks.

Results: We included 193 patients, mostly men, 171 men (81.9\%), with a mean age of $47.94 \pm 12,04$ years and a mean disease duration of 20.07 \pm 11.18 years. At the moment of the evaluation $49.7 \%$ were still employed and $50.3 \%$ were retired due to work disability. Most patients had a professional qualification (89.2\%), and manual labor was identified in 132 $(6 ., 4 \%)$ of the patients. In the study cohort the professional physical strain was mostly low (39.4\%) and medium (50.8\%). Manual laborers $(77.3 \%)$ had an active disease, with a mean BASDAl of $5.67(\mathrm{p}=0.006)$ and a mean ASDAS-PCR of $3.2 \pm 1.23$, the high and very high disease activity (ASDAS-PCR $>2.1$ ) being registered in $86.1 \%$ of them $(p<0.001)$. Manual workers had an important functional deficit (BASFI=6.32 \pm 2.41 ) $(p=0,001)$. Regarding radiological features, manual laborers had stage IV sacroilitis in $68.9 \%$ of the cases, $82.6 \%$ had syndesmophytes, which were generalised in $59.8 \%$ of the cases $(p=0.005)$.

A high professional physical strain correlates with active disease (BAS$\mathrm{DAl}=6.22)$, with the presence of bridging syndesmophytes $(81.8 \%$, $\mathrm{p}=0.009)$, and generalized syndesmophytes $(68.8 \%, \mathrm{p}=0.007)$.

Conclusion: Manual labor, with strenuous physical activities correlates with high disease activity, severe functional impairment and radiological changes in AS patients.

\section{REFERENCES:}

[1]

Jacques, Peggy, et al. "Proof of concept: enthesitis and new bone formation in spondyloarthritis are driven by mechanical strain and stromal cells." Annals of the rheumatic diseases73.2 (2014): 437-445.

[2] Ramiro, Sofia, et al. "Lifestyle factors may modify the effect of disease activity on radiographic progression in patients with ankylosing spondylitis: a longitudinal analysis." RMD open 1.1 (2015): e000153.

[3] 3. Sirjita N. Elemente de expertiză medicală şi recuperarea capacității de muncă. Editura TipArg, 2004 pp 112-118

Disclosure of Interests: None declared DOI: 10.1136/annrheumdis-2019-eular.8062

\section{THU0369} INCREASED PREVALENCE OF CARDIAC DISORDERS IN DUTCH ANKYLOSING SPONDYLITIS PATIENTS: THE CARDAS STUDY

Milad Baniaamam ${ }^{1}$, Sjoerd C. Heslinga ${ }^{2}$, Thelma Konings ${ }^{3}$, Otto Kamp ${ }^{3}$, Vokko P. van Halm ${ }^{3}$, Irene van der Horst-Bruinsma ${ }^{2}$, Michael Nurmohamed ${ }^{1,2}$.

${ }^{1}$ Amsterdam Rheumatology and immunology Center, location Reade, Department of Rheumatology, Amsterdam, Netherlands; ${ }^{2}$ Amsterdam Rheumatology and immunology Center, location VUmc, Department of Rheumatology, Amsterdam, Netherlands; ${ }^{3}$ Amsterdam University Medical Center, location VUmc, Department of Cardiology, Amsterdam, Netherlands

Background: The overall mortality rate in ankylosing spondylitis (AS) patients is increased by $60-90 \%$ compared with the general population. This higher mortality rate is predominately caused by cardiovascular disease (CVD) comprising both by increased prevalence of cardiac diseases such as valvular heart disease, conduction disturbances and cardiomyopathies as well as atherosclerotic diseases such as myocardial infarctions. However, there is some diversity in the literature and there is a lack of contemporary studies. Therefore, we investigated current prevalences of cardiac disorders in a well characterized cohorts of Dutch patients with AS

Objectives: The CARDAS study aims to describe the prevalence of CVD in AS patients in a Dutch cohort.

Methods: We performed a cross-sectional study in consecutive AS patients between 50-75 years. Subjects were recruited from a large rheumatology outpatient clinic (Reade) in Amsterdam, the Netherlands. Patients underwent echocardiography with 2D, spectral and Color Doppler imaging. Diastolic dysfunction was evaluated by an experienced cardiologist based on the 2008 ASE guideline. Furthermore, an ECG, blood sample, surveys and physical examination were performed. Disease activity and function were measured with the BASFI, BASDAI and the ASDASCRP

Results: 191 Consecutive AS patients were included with a median age of 58 years (54-65) of which mostly men $(136 / 191,71 \%)$ (Table 1). The mean disease duration was 34.9 years $( \pm 11.9)$ The disease activity measures, BASDAI, ASDAS-CRP and BASFI, indicated moderate disease activity and were, respectively $3.1( \pm 2,3), 2.1( \pm 1.0)$ and $3.5(1.6-5.7)$ The use of bDMARD's was present in $42 \%$ of the AS patients. As cardiac manifestations are age related, AS patients were divided in 3 age categories, respectively, 50-59 years, 60-69 years and 70-75 years Hypertension was diagnosed in respectively $45.3 \%, 64.1 \%$ and $85.7 \%$. History of CVD described as angina pectoris, myocardial infarction, stroke and/or peripheral ischemia were present in respectively $7.5 \%, 9.4 \%$ and $19 \%$. Diastolic dysfunction was present in respectively $40.4 \%, 61.9 \%$ and $89.5 \%$ of the AS patients. The diastolic dysfunction present in this population was mainly mild with $60.2 \%$ of patients presenting with grade 1 and $39.8 \%$ with grade 2 diastolic dysfunction. Moderate to severe aortic regurgitation was present in, respectively $10.4 \%, 12.5 \%$ and $33.4 \%$ and moderate to severe mitral valve regurgitation in $33 \%, 31.3 \%$ and $42.9 \%$ of the AS patients. These prevalences are substantially increased in comparison to the general Dutch population.

Conclusion: We demonstrated increased prevalences of diastolic dysfunction, aortic valve regurgitation, mitral valve regurgitation and hypertension in Dutch AS patients compared to age matched general population Although our results suggest mandatory echocardiography screening, this first needs to be established in prospective follow-up studies.

Abstract THU0369 - Table 1. Patient characteristics

\begin{tabular}{lcccc}
\hline & All & $\mathbf{5 0 - 5 9}$ years & $\mathbf{6 0 - 6 9}$ years & $\mathbf{7 0 - 7 5}$ years \\
\hline $\mathbf{N}$ & 191 & & & \\
Men & $131(71 \%)$ & & & \\
Age (years) & $58(54-65)$ & & & \\
Disease activity & & & & \\
BASDAI & $3.1( \pm 2,3)$ & & & \\
ASDAS-CRP & $2.1( \pm 1.0)$ & & & \\
BASFI & $3.5(1.6-5.7)$ & & $9.4 \%$ & $19 \%$ \\
CVD & & $7.5 \%$ & $64.1 \%$ & $85.7 \%$ \\
History of CVD* & & $45.3 \%$ & $12.5 \%$ & $33.4 \%$ \\
Hypertension & & $10.4 \%$ & $31.1 \%$ & $42.9 \%$ \\
Aortic valve regurgitation ${ }^{\star *}$ & & $33 \%$ & $61.9 \%$ & $89.5 \%$ \\
Mitral valve regurgitation & & & & \\
Diastolic dysfunction & & $40.4 \%$ & & \\
\hline
\end{tabular}

* Described as angina pectoris, myocardial infarction, stroke and/or peripheral ischemia

** moderate - severe regurgitation.

Disclosure of Interests: Milad Baniaamam: None declared, Sjoerd C Heslinga: None declared, Thelma Konings: None declared, Otto Kamp: None declared, Vokko P. van Halm: None declared, Irene van der HorstBruinsma Grant/research support from: MSD, Pfizer, AbbVie, Consultant 
for: Abbvie, UCB, MSD, Novartis, Speakers bureau: BMS, AbbVie, Pfizer, MSD, Michael Nurmohamed Grant/research support from: AbbVie, BristolMyers Squibb, Celgene, Eli Lilly, Janssen, Menarini, MSD, Mundipharma, Pfizer, Roche, Sanofi and UCB, Consultant for: AbbVie, Bristol-Myers Squibb, Celgene, Eli Lilly, Janssen, Menarini, MSD, Mundipharma, Pfizer, Roche, Sanofi and UCB, Speakers bureau: AbbVie, Bristol-Myers Squibb, Celgene, Eli Lilly, Janssen, Menarini, MSD, Mundipharma, Pfizer, Roche, Sanofi and UCB

DOI: 10.1136/annrheumdis-2019-eular.5703

\section{THU0370 PERFORMANCE OF THE ANKYLOSING SPONDYLITIS DISEASE ACTIVITY SCORE (ASDAS) IN PATIENTS WITH PERIPHERAL AND AXIAL SPONDYLOARTHRITIS IN CLINICAL PRACTICE}

Esther Beckers ${ }^{1}$, Marin Been ${ }^{1}$, Casper Webers ${ }^{1}$, Annelies Boonen ${ }^{1}$, Peter Ten Klooster ${ }^{2}$, Harald Vonkeman ${ }^{2}$, Astrid van Tubergen ${ }^{1} .{ }^{1}$ MUMC, Rheumatology, Maastricht, Netherlands, ${ }^{2} \mathrm{MST}$, Rheumatology, Enschede, Netherlands

Background: The Ankylosing Spondylitis Disease Activity Score (ASDAS) is a widely used composite measure of disease activity in axial spondyloarthritis $(a x S p A)$. Such a tool is lacking for peripheral SpA (pSpA). However, the ASDAS also assesses peripheral joint symptoms. Previously, trial data have shown that the ASDAS has high sensitivity to change and high discriminatory ability between disease states in $\mathrm{PSpA}$ (1). We hypothesized that the ASDAS can be used to measure disease activity in all patients with $\mathrm{pSpA}$ in clinical practice.

Objectives: To investigate the construct validity of the ASDAS in pSpA in comparison with axSpA in clinical practice.

Methods: Data from a registry for SpA in the Netherlands (SpA-Net) were used. Construct validity of the ASDAS was assessed by testing hypotheses about Spearman correlations with other outcomes measures including individual components of disease activity, physical functioning and health-related quality of life (HRQoL). Construct validity was also assessed by stratifying patients according to ASDAS cut-offs to compare means of different health outcomes across ASDAS states by one-way ANOVA analyses. All analyses were repeated after stratification for the presence/absence of psoriasis in $\mathrm{pSpA}$. Results were compared to the performance of the ASDAS in axSpA.

Results: In total, 194 patients with pSpA and 222 patients with axSpA were included. Poor to high correlation was found between ASDAS and measures of disease activity $\left(\mathrm{r}_{\mathrm{s}} 0.20\right.$ to 0.78$)$, a moderate correlation was found between ASDAS and measures of physical functioning $\left(r_{s} 0.61\right.$ to 0.64 ) and a poor to moderate correlation was found between ASDAS and measures of $\mathrm{HRQoL}$ in PSpA $\left(\mathrm{r}_{\mathrm{s}}-0.27\right.$ to -0.66$)$ (table 1). With increasing ASDAS states, significantly worse scores were found on (other) measures of disease activity, physical functioning and HRQoL in $\mathrm{pSpA}$ (table 2). Stratification for the presence/absence of psoriasis showed similar results (data not shown). All results for $\mathrm{pSpA}$ were comparable to axSpA (see for example Table 1).

Abstract THU0370 - Table 1. Construct validity of ASDAS with health outcomes in pSpA and axSpA

\begin{tabular}{lcc}
\hline & pSpA $\mathbf{n = 1 9 4}$ & axSpA $\mathbf{n = 2 2 2}$ \\
\hline Disease activity & & \\
\hline BASDAl & 0.78 & 0.81 \\
Patient global & 0.77 & 0.75 \\
CRP & 0.41 & 0.51 \\
VAS pain & 0.73 & 0.67 \\
Physician global & 0.55 & 0.46 \\
TJC68 & 0.38 & 0.28 \\
SJC66 & 0.20 & 0.24 \\
Physical functioning & & \\
BASFI & 0.61 & 0.59 \\
HAQ-S & 0.62 & 0.58 \\
ASAS-HI & 0.64 & 0.46 \\
Quality of life & & \\
EQ5D & -0.56 & -0.40 \\
SF36 MCS & -0.27 & -0.26 \\
SF36 PCS & -0.66 & -0.54 \\
All correlations were statistically significant $(p<0.05)$ &
\end{tabular}

Criteria for correlations: $r_{\mathrm{s}}<0.29$ poor, $0.30 \leq r_{\mathrm{s}}<0.49$ low, $0.50 \leq r_{\mathrm{s}}<0.69$ moderate, 0.70 $\leq r_{\mathrm{s}}<0.89$ high and $r_{\mathrm{s}} \geq 0.90$ very high

Abstract THU0370 - Table 2. Health outcomes according to ASDAS states in pSpA

\begin{tabular}{lccccc}
\hline ASDAS cut-off & $<\mathbf{1 . 3}$ & $\mathbf{1 . 3}$ to $<2.1$ & $\mathbf{2 . 1}$ to $\leq \mathbf{3 . 5}$ & $>\mathbf{3 . 5}$ & p-value \\
Disease activity & $\mathbf{n}=\mathbf{4 0}$ & $\mathbf{n}=\mathbf{5 9}$ & $\mathbf{n}=\mathbf{8 1}$ & $\mathbf{n}=\mathbf{1 4}$ & \\
\hline BASDAI & $1.6(1.0)$ & $2.9(1.4)$ & $5.1(1.8)$ & $7.1(1.3)$ & $<0.01$
\end{tabular}

\begin{tabular}{lccccc} 
Patient global & $1.2(1.1)$ & $2.7(1.7)$ & $5.0(2.1)$ & $7.4(1.9)$ & $<0.01$ \\
CRP & $1.7(1.3)$ & $3.4(3.6)$ & $5.4(7.3)$ & $9.0(7.2)$ & $<0.01$ \\
VAS pain & $1.1(1.6)$ & $2.6(2.3)$ & $5.3(2.2)$ & $6.5(1.3)$ & $<0.01$ \\
Physician global & $0.9(0.9)$ & $1.2(0.1)$ & $2.4(1.8)$ & $4.4(1.6)$ & $<0.01$ \\
TJC68 & $0.2(0.4)$ & $1.2(3.8)$ & $2.1(4.0)$ & $2.6(2.5)$ & $<0.05$ \\
SJC66 & $0.1(0.4)$ & $0.4(1.0)$ & $0.5(1.2)$ & $0.6(0.9)$ & 0.29 \\
Physical functioning & & & & & \\
BASFI & $1.1(1.4)$ & $2.1(2.0)$ & $4.0(2.2)$ & $5.4(2.4)$ & $<0.01$ \\
HAQ-S & $0.3(0.3)$ & $0.7(0.6)$ & $1.0(0.6)$ & $1.5(0.8)$ & $<0.01$ \\
ASAS-HI & $2.8(2.7)$ & $4.6(3.1)$ & $7.0(3.2)$ & $9.4(1.8)$ & $<0.01$ \\
HRQoL & & & & & \\
EQ5D & $0.9(0.1)$ & $0.8(0.1)$ & $0.7(0.2)$ & $0.6(0.3)$ & $<0.01$ \\
SF36 MCS & $52.8(8.1)$ & $52.7(9.7)$ & $47.1(10.7)$ & $41.5(14.1)$ & $<0.01$ \\
SF36 PCS & $48.9(8.3)$ & $40.6(10.7)$ & $35.1(7.6)$ & $29.5(5.6)$ & $<0.01$ \\
Values are expressed as mean (SD) & & & & \\
\hline
\end{tabular}

Conclusion: The ASDAS demonstrated similar construct validity in all patients with $\mathrm{pSpA}$ and axSpA in clinical practice. Therefore, the ASDAS can also be used to measure disease activity in $\mathrm{pSpA}$ in daily practice.

\section{REFERENCE:}

[1] Turina MC, et al. A psychometric analysis of outcome measures in peripheral spondyloarthritis. ARD 2016;75:1302-7.

Disclosure of Interests: None declared

DOI: 10.1136/annrheumdis-2019-eular.1617

\section{THU0371 PREVALENCE OF WORK DISABILITY AND PREDICTORS OF WORK PRODUCTIVITY AMONG EMPLOYABLE PATIENTS WITH ANKYLOSING SPONDYLITIS AND PSORIATIC ARTHRITIS IN A CANADIAN REAL WORLD OBSERVATIONAL COHORT INTERIM RESULTS FROM THE COMPLETE STUDIES}

Louis Bessette $^{1}$, Valencia P. Remple ${ }^{2}$, Samuel Silverberg ${ }^{3}$, Viktoria Pavlova ${ }^{4}$, Majed Khraishi'. 'Laval University, Centre Hospitalier de l'Université Laval, Quebec, Canada: ${ }^{2}$ AbbVie Corporation, Montreal, Canada; ${ }^{3}$ Etobicoke General Hospital, Toronto, Canada; ${ }^{4}$ McMaster University, Hamilton, Canada; ${ }^{5}$ Memorial University of Newfoundland, St. John's, Canada

Background: Work disability is an important functional outcome among patients with chronic inflammatory diseases such as ankylosing spondylitis (AS) and psoriatic arthritis (PsA). Maintenance of patients in the work force and return to employment are important treatment outcomes with implications for both patients and the healthcare system.

Objectives: The aim of this analysis is to describe the prevalence of unemployment due to disability at baseline and to identify factors associated with work productivity loss in patients with AS and PsA followed in Canadian routine clinical care.

Methods: Patients eligible for the COMPLETE studies were anti-TNFo naïve adults, with active AS or PsA per the judgment of the treating physician, who required change in their treatment regimen. This interim analysis included patients that were treated with adalimumab or non-biologic DMARDs and, were either employed or on disability at baseline. Work productivity was measured by the Work Limitations Questionnaire (WLQ). Depression was defined as a Beck's Depression Inventory (BDI) score $\geq 20$ and/or treatment with an antidepressant/anxiolytic at baseline. Multivariate generalized linear models were used to identify determinants of\% WLQ productivity loss (WLQ-PL) scores at 6 and 12 months of treatment, along with the respective changes from baseline. Least Square Means (LSM) for the WLQ-PL improvement were reported from the multivariate model.

Results: A total of 486 AS patients and 292 PsA patients were included in the analysis. The mean (SD) disease duration was 5.2 (8.6) and 12.8 (12.1) years, mean (SD) age was 41.7 (11.6) and 48.3 (10.5) years, and male predominance was $58.4 \%$ and $54.5 \%$, in the AS and PsA groups, respectively. At baseline, $13.4 \%$ of AS patients and $17.8 \%$ of PsA patients were unemployed due to disability.

Among employed patients, the mean (SD) WLQ-PL score at baseline was $9.2 \%$ (5.7) in the AS patient group and $8.3 \%$ (6.0) in the PsA patient group. After 6 months of treatment significant improvement was observed in both patient populations $\left(\Delta_{\mathrm{AS}}[95 \% \mathrm{Cl}]\right.$ : $-2.7 \% \quad[-3.4,-2.0]$ $\Delta_{\text {PsA }}[95 \%$ Cl] : $-2.1 \%[-2.9,-1.3])$ which was maintained until 12 months. Among AS patients, after adjusting for baseline parameters including age sex, tobacco use, HLA B27 status, treatment group, depression, and baseline scores for BASFI and WLQ-PL using multivariate analysis, presence of depression (LSM: $-0.2 \%$ vs. $-2.5 \% ; \mathrm{p}=0.016$ ) and female sex (LSM: $-0.7 \%$ vs. $-2.0 \% ; p=0.047$ ) were identified as significant negative 